\title{
SOME ASPECTS OF UNIQUENESS FOR SOLUTIONS TO BOUNDARY PROBLEMS $\dagger$
}

\author{
by M. H. MARTIN \\ (Received 9th August, 1961)
}

\section{Introduction}

The solution to the boundary problem

$$
\Delta u=u_{x x}+u_{y y}=0 \text { in } r \leqq 1, u_{r}=h u \text { on } r=1,
$$

where $r$ is the distance of point $(x, y)$ from the origin, and $h$ is a given function of the arc length $s$ along the unit circle $r=1$, is not necessarily unique, Boggio (1), Weinstein (2), Stoker (3), Martin (4). Indeed if $h$ is a positive integer $m$, it is known that the only solutions regular analytic for $r \leqq 1$ are

$$
u=A r^{m} \cos m \theta+B r^{m} \sin m \theta,
$$

where $r, \theta$ denote polar coordinates and $A, B$ are arbitrary constants.

On the other hand it is easy to see that if the ratio $\lambda=u_{1} / u_{2}$ of two of these solutions $u_{1}, u_{2}$ is required to be regular analytic in $r \leqq 1$, the two solutions must be linearly dependent. This example shows that even though the solution of the boundary problem is not unique, the imposition of a further hypothesis implies linear dependence between two solutions.

The problem considered in (4) and in the present paper follows naturally from this example and is formulated as follows.

What can be said about the uniqueness of a function $u$, harmonic in a region $S$, if along the boundary $C$ of $S$ the external normal derivative $u_{n}$ is a prescribed separable function

$$
u_{n}=h(s) f(u)
$$

of $u$ and the arc length $s$ of $C$ ?

In this paper we approach the problem from a new point of view and extend earlier results (4). We assume that $S$ is a simply connected region bounded by a single analytic curve $C$, and that $S$ lies in the interior of a region $R$ within which $u$ is regular analytic. The functions $h(s), f(u)$ are real functions and are assumed regular analytic for all real values of their arguments.

If $u_{0}$ is a zero of $f(u)$, an obvious solution is the constant solution $u=u_{0}$. Such trivial solutions are disregarded and only non-constant solutions considered.

† This work was supported by the Guggenheim Memorial Foundation and by the United States Air Force through the Air Force Office of Scientific Research of the Air Research and Development Command under Contract No. AF 49(638)-570 with the University of Maryland. 


\section{Preliminary Considerations}

We begin with the formula

$$
\int_{c} \tau\left(f_{2} \frac{\partial u_{1}}{\partial n}-f_{1} \frac{\partial u_{2}}{\partial n}\right) d s=\int_{S}\left[a p_{1}^{2}+2 b p_{1} p_{2}+c p_{2}^{2}+a q_{1}^{2}+2 b q_{1} q_{2}+c q_{2}^{2}\right] d S
$$

valid for any pair of functions $u_{1}, u_{2}$ harmonic in $R \supset S$. Here we have written

and $\tau=\tau\left(u_{1}, u_{2}\right)$ with

$$
f_{1}=f\left(u_{1}\right), \quad f_{2}=f\left(u_{2}\right), \quad p_{i}=\frac{\partial u_{i}}{\partial x}, \quad q_{i}=\frac{\partial u_{i}}{\partial y}, \quad(i=1,2),
$$

$$
a=f_{2} \tau_{u_{1}}, \quad 2 b=f_{2} \tau_{u_{2}}-f_{1} \tau_{u_{1}}+\left(f_{2}^{\prime}-f_{1}^{\prime}\right) \tau, \quad c=-f_{1} \tau_{u_{2}},
$$

where the primes denote differentiations. The formal verification of (2.1) for an arbitrary function $\tau$ is an easy application of Gauss's theorem, but for the formula to be valid, the function $\tau$ and the functions $u_{1}, u_{2}$ must be chosen so that the integrals in (2.1) exist.

To bring out the connection of (2.1) with the uniqueness question (I), suppose a real function $\tau$ subject to the conditions.

$$
b^{2}-a c=0, \quad a>0,
$$

can be found for which the integrals in (2.1) exist for a given pair of harmonic functions $u_{1}, u_{2}$. In this event (2.1) may be given the form

$\int_{C} \tau\left(f_{2} \frac{\partial u_{1}}{\partial n}-f_{1} \frac{\partial u_{2}}{\partial n}\right) d s=\int_{S}\left[\left(\sqrt{a} p_{1}+\sqrt{c} p_{2}\right)^{2}+\left(\sqrt{a} q_{1}+\sqrt{c} q_{2}\right)^{2}\right] d S . .$.

If both $u_{1}, u_{2}$ satisfy the boundary condition (I), the integral around $C$ vanishes and the vanishing of the integral over $S$ implies that the equations

$$
\sqrt{a} p_{1}+\sqrt{c} p_{2}=0, \quad \sqrt{a} q_{1}+\sqrt{c} q_{2}=0
$$

hold everywhere in $S$. As a consequence the Jacobian of $u_{1}, u_{2}$ vanishes identically in $S$ and therefore $u_{1}, u_{2}$ are functionally dependent.

At this point the following lemma applies.

Lemma 2.1. If two harmonic functions $u_{1}, u_{2}$ are functionally dependent, they are linearly related, i.e., real constants $k$, l exist for which

$$
u_{2}=k u_{1}+l \text {. }
$$

The proof of this lemma is easy and is omitted.

Thus the two solutions $u_{1}, u_{2}$ must be linearly related, i.e. satisfy (2.6). Clearly this implies that

$$
\frac{\partial u_{2}}{\partial n}=k \frac{\partial u_{1}}{\partial n} \text { on } C,
$$

and therefore $f\left(u_{2}\right)=k f\left(u_{1}\right)$ so that $f(u)$ necessarily satisfies the functional equation

$$
f\left(k u_{1}+l\right)=k f\left(u_{1}\right)
$$


Recalling that we have laid aside constant solutions, we digress a moment to prove the following lemma.

Lemma 2.2. The only real, non-constant, analytic solutions of the functional equation

$$
f(k u+l)=k f(u), \quad k, l=\text { const. (real) }
$$

are the linear functions

$$
f=m\left(u-u_{0}\right), \quad u_{0}=l /(1-k), \quad m=\text { const. (arbitrary), }
$$

if $k \neq \pm 1$. If $k=1$, the functional equation defines the periodic functions of period $l$; if $k=-1$, it defines the odd functions of $u-l / 2$.

Only the statement for $k \neq \pm 1$ requires proof. Differentiating (2.8) $n$ times and then placing $u=u_{0}$, we obtain

$$
\left(k^{n-1}-1\right) f^{(n)}\left(u_{0}\right)=0, \quad n=0,1,2, \ldots,
$$

inasmuch as $k u_{0}+l=u_{0}$. Therefore $f$ and all its derivatives vanish at $u=u_{0}$, except the first which remains arbitrary. Consequently the Taylor series expansion of $f(u)$ in powers of $u-u_{0}$ reduces to $f=m\left(u-u_{0}\right)$, with $m$ an arbitrary constant. Conversely it is easy to verify that $f=m\left(u-u_{0}\right)$ is a solution of the functional equation for arbitrary constant $m$, provided $u_{0}$ is as given. This completes the proof of the lemma which will be used in the proof of Theorem 2.1 below.

If $f(u)$ is linear in $u$, say $f(u)=m\left(u-u_{0}\right)$ the functional equation (2.7) requires that $l=(1-k) u_{0}$ in (2.6) to yield $u_{2}-u_{0}=k\left(u_{1}-u_{0}\right)$. Thus the differences $u_{2}-u_{0}, u_{1}-u_{0}$ are linearly dependent.

If $f(u)$ is not linear in $u$, it follows from Lemma 2.2 that either $k=+1$, in which case $f(u)$ is periodic of period $l$ and $u_{2}-u_{1}=l$ from (2.6); or $k=-1$, whereupon $f(u)$ is an odd function of $u-u_{0}$ and (2.6) requires $u_{1}+u_{2}=2 u_{0}$.

If $f(u)$ is not linear, periodic, nor an odd function of $u-u_{0}$, the functional equation (2.7) can hold only if $k=1, l=0$, and therefore $u_{1}=u_{2}$ from (2.6). A non-constant solution $u_{1}$ is therefore unique.

Summing up our results, we have the theorem

Theorem 2.1. If $u_{1}, u_{2}$ are two non-constant solutions of the boundary problem

$$
\Delta u=0 \text { in } S, \quad u_{n}=h(s) f(u) \text { on } C,
$$

regular analytic in $R \supset S$, and a function $\tau=\tau\left(u_{1}, u_{2}\right)$ can be found subject to the conditions (2.4) such that (2.5) is valid, the two solutions $u_{1}, u_{2}$ must be linearly related, i.e., $u_{2}=k u_{1}+l$.

If $f(u)$ is linear, say $f=m\left(u-u_{0}\right)$, the differences $u_{1}-u_{0}, u_{2}-u_{0}$ are linearly dependent. If $f(u)$ is not linear, the two solutions $u_{1}, u_{2}$ must be identical unless $f(u)$ is periodic or an odd function of $u-u_{0}$, whereupon either the difference, or the sum of $u_{1}, u_{2}$, is constant respectively. 
The first condition in (2.4) is a non-linear partial differential equation

$$
\left[f_{1} \tau_{u_{1}}-f_{2} \tau_{u_{2}}+\left(f_{1}^{\prime}-f_{2}^{\prime}\right) \tau\right]^{2}+4 f_{1} f_{2} \tau_{u_{1}} \tau_{u_{2}}=0
$$

for $\tau$, and the second imposes the inequality $a=f_{2} \tau_{u_{1}}>0$.

\section{The Function $\tau$}

If we set

$$
T=\log \tau
$$

the partial differential equation (2.9) for $\tau$ becomes

$$
\left(f_{1} T_{u_{1}}-f_{2} T_{u_{2}}+f_{1}^{\prime}-f_{2}^{\prime}\right)^{2}+4 f_{1} f_{2} T_{u_{1}} T_{u_{2}}=0 .
$$

This is the equation of a parabola in the plane of the variables $T_{u_{1}}, T_{u_{2}}$. If we introduce a uniformising parameter $t$ by placing

$$
T_{u_{1}}=-\frac{\left(t-f_{1}^{\prime}\right)^{2}}{f_{1}\left(f_{1}^{\prime}-f_{2}^{\prime}\right)}, \quad T_{u_{2}}=\frac{\left(t-f_{2}^{\prime}\right)^{2}}{f_{2}\left(f_{1}^{\prime}-f_{2}^{\prime}\right)},
$$

the integrability condition yields a quasi-linear partial differential equation

$$
f_{1}\left(t-f_{2}^{\prime}\right) t_{u_{1}}+f_{2}\left(t-f_{1}^{\prime}\right) t_{u_{2}}=\frac{f_{1} f_{1}^{\prime \prime}\left(t-f_{2}^{\prime}\right)^{2}-f_{2} f_{2}^{\prime \prime}\left(t-f_{1}^{\prime}\right)^{2}}{2\left(f_{1}^{\prime}-f_{2}^{\prime}\right)}
$$

for $t$. Corresponding to a solution $t$ of this equation we obtain a solution $\tau$ of (2.9) from

$$
\tau=C \exp \int\left\{T_{u_{1}} d u_{1}+T_{u_{2}} d u_{2}\right\}, \quad C=\text { const., }
$$

for $T_{u_{1}}, T_{u_{2}}$ as given in (3.3).

The quantities $a, b, c$ defined in (2.3) take the following forms

$$
a=-\frac{f_{2}}{f_{1}} \frac{\left(t-f_{1}^{\prime}\right)^{2}}{f_{1}^{\prime}-f_{2}^{\prime}} \tau, \quad b=\frac{\left(t-f_{1}^{\prime}\right)\left(t-f_{2}^{\prime}\right)}{f_{1}^{\prime}-f_{2}^{\prime}} \tau, \quad c=-\frac{f_{1}}{f_{2}} \frac{\left(t-f_{2}^{\prime}\right)^{2}}{f_{1}^{\prime}-f_{2}^{\prime}} \tau
$$

The requirement $b^{2}-a c=0$ in (2.4) is obviously fulfilled. If $f_{1} \neq 0,,_{2} \neq 0$, $f_{1}^{\prime} \neq f_{2}^{\prime}$ hold in $R$, it follows from (3.3) and (3.5) that $\tau$ is regular analytic in $R$ and maintains a fixed sign. From (3.6) it follows that the second requirement $a>0$ in (2.4) may now be met by adjusting the arbitrary constant $C$ in $\tau$.

Thus we arrive at the following lemma.

Lemma 3.1. A function $\tau$ fulfilling the hypotheses of Theorem 2.1 can be found and the conclusions of the theorem apply, provided

$$
f_{1} \neq 0, \quad f_{2} \neq 0, \quad f_{1}^{\prime} \neq f_{2}^{\prime},
$$

hold in $R \supset S$ and a solution $t=t\left(u_{1}, u_{2}\right)$ of (3.4) regular analytic in $R$ is at hand.

In view of this lemma, Theorem 2.1 yields

Theorem 3.1. If $u_{1}, u_{2}$ are two non-constant solutions of the boundary problem

$$
\Delta u=0 \text { in } S, \quad u_{n}=h(s) f(u) \text { on } C,
$$


regular analytic in $R \supset S$ such that

$$
f_{1} \neq 0, f_{2} \neq 0, f_{1}^{\prime} \neq f_{2}^{\prime}
$$

hold in $R$ and a solution $t=t\left(u_{1}, u_{2}\right)$ of (3.4) regular analytic in $R$ can be found, the two solutions $u_{1}, u_{2}$ must be linearly related, i.e., $u_{2}=k u_{1}+l$.

If $f(u)$ is linear, say $f=m\left(u-u_{0}\right)$, the differences $u_{1}-u_{0}, u_{2}-u_{0}$ are linearly dependent. If $f(u)$ is not linear, the two solutions $u_{1}, u_{2}$ must be identical unless $f(u)$ is periodic or an odd function $u-u_{0}$, whereupon either the difference or the sum of $u_{1}, u_{2}$ is constant respectively.

When new independent variables

$$
\xi=\int \frac{d u_{1}}{f_{1}}-\int \frac{d u_{2}}{f_{2}}, \quad \eta=\int \frac{f_{1}^{\prime}}{f_{1}} d u_{1}-\int \frac{f_{2}^{\prime}}{f_{2}} d u_{2},
$$

are introduced into (3.4) this equation takes a normal form

where

$$
t_{\xi}+t t_{\eta}=K t^{2}+2 L t+M
$$

$$
K=\frac{f_{1} f_{1}^{\prime \prime}-f_{2} f_{2}^{\prime \prime}}{2\left(f_{1}^{\prime}-f_{2}^{\prime}\right)^{2}}, \quad L=-\frac{f_{1} f_{1}^{\prime \prime} f_{2}^{\prime}-f_{1}^{\prime} f_{2} f_{2}^{\prime \prime}}{2\left(f_{1}^{\prime}-f_{2}^{\prime}\right)^{2}}, \quad M=\frac{f_{1} f_{1}^{\prime \prime} f_{2}^{\prime 2}-f_{1}^{\prime 2} f_{2} f_{2}^{\prime \prime}}{2\left(f_{1}^{\prime}-f_{2}^{\prime}\right)^{2}}, \ldots
$$

and we find, from (3.3) that

$$
T_{\xi}=\frac{f_{1}^{\prime} f_{2}^{\prime}-t^{2}}{f_{1}^{\prime}-f_{2}^{\prime}}, \quad T_{\eta}=\frac{2 t-f_{1}^{\prime}-f_{2}^{\prime}}{f_{1}^{\prime}-f_{2}^{\prime}}
$$

Clearly the integration of (3.9) is an important step, and we turn to this in the next section.

\section{The Uniformising Parameter $t$}

In some cases the partial differential equation (3.9) for $t$ integrates by separation of variables. When this is not the case, the lemmas below sometimes apply.

Lemma 4.1. The nonlinear partial differential equation

$$
t_{\xi}+t t_{\eta}=K t^{2}+2 L t+M
$$

will have solutions in common with the linear equation

provided

$$
t_{\eta}-K t=L+X(\xi)
$$

$$
K_{\xi}=L_{\eta}, \quad L_{\xi}-M_{\eta}+K M-L^{2}=F(\xi),
$$

and $X(\xi)$ is a solution of the Ricatti equation

$$
X^{\prime}+X^{2}+F(\xi)=0 .
$$

Corresponding to each solution $X(\xi)$ of this equation, equations (4.1), (4.2) share a one-parameter family of solutions

$$
t=e^{J} \int\left\{M e^{-J} d \xi+(L+X) e^{-J} d \eta\right\}+C e^{J}, \quad C=\text { const. }
$$


where

$$
J=\int\{(L-X) d \xi+K d \eta\}
$$

Solving (4.1), (4.2) simultaneously for $t_{\xi}, t_{\eta}$ we find

$$
t_{\xi}=(L-X) t+M, \quad t_{\eta}=K t+L+X .
$$

A simple calculation shows that the integrability condition $t_{\xi \eta}=t_{\eta \xi}$ is satisfied identically if (4.3), (4.4) hold. Conversely if these hold, the line integral (4.6) defines a function $J=J(\xi, \eta)$ and $e^{-J}$ serves as a simultaneous integrating factor for the linear equations (4.7), for they may be written

$$
\frac{\partial}{\partial \xi} e^{-J} t=e^{-J} M, \quad \frac{\partial}{\partial \eta} e^{-J} t=e^{-J}(L+X)
$$

and the integrability condition arising from these equations is satisfied identically. Consequently (4.5) offers a one parameter family of simultaneous solutions to the linear equations (4.7) and, since these equations imply (4.1), (4.2), the lemma is proved.

This lemma forms the basis for the next one.

Lemma 4.2. The non-linear partial differential equation

$$
t_{\xi}+t t_{\eta}=w_{\eta} t^{2}+2 w_{\xi} t+w_{\eta}, \quad w=w(\xi, \eta),
$$

has a one-parameter family of solutions

$$
t=\frac{C-Z H_{\xi}+H Z^{\prime}}{Q Z}, \quad H=H(\xi, \eta)=\int_{0}^{\eta} Q(\xi, \eta) d \eta, \quad C=\text { const., }
$$

corresponding to each solution $Z=Z(\xi)$ of the linear equation

$$
Z^{\prime \prime}+F(\xi) Z=0
$$

provided the function $Q=e^{-w}$ meets the conditions

$$
Q_{\xi \xi}-Q_{\eta \eta}+F(\xi) Q=0, \quad Q_{\eta}(\xi, 0) \equiv 0
$$

If we identify

$$
K=M=w_{\eta}, \quad L=w_{\xi},
$$

in (4.1) the first condition in (4.3) is obviously satisfied, and the second reduces to

or to

$$
w_{\xi \xi}-w_{\eta \eta}-\left(w_{\xi}^{2}-w_{\eta}^{2}\right)=F(\xi)
$$

$$
Q_{\xi \xi}-Q_{\eta \eta}+F(\xi) Q=0 \text { if } w=-\log Q,
$$

and the Ricatti equation (4.4) is replaced by the linear equation (4.10) if we employ the well-known transformation

$$
X=\frac{Z^{\prime}}{Z}
$$

Substituting from (4.12), (4.13) into (4.6) we find

$$
J=-\log Q Z
$$


and consequently from (4.5) that

$$
t=\frac{C-I}{Q Z}, \quad I=\int\left\{Z Q_{\eta} d \xi+\left(Z Q_{\xi}-Q Z^{\prime}\right) d \eta\right\} .
$$

If we introduce the function $H$ defined in (4.9) the line integral $I$ reduces to

$$
I=Z H_{\xi}-H Z^{\prime},
$$

provided (4.11) hold. To verify this we need only show that $I_{\xi}=Z Q_{\eta}$, since $I_{\eta}=Z Q_{\xi}-Q Z^{\prime}$ is obvious from the definition of $H$. Calculating $I_{\xi}$ from (4.14), we find, by virtue of (4.11) that

$$
I_{\xi}=Z H_{\xi \xi}-H Z^{\prime \prime}=Z \int_{0}^{n} Q_{\eta \eta} d \eta=Z Q_{n} .
$$

The solutions (4.5) and (4.9) involve two arbitrary constants and therefore constitute complete solutions. They may be used to construct the general solutions of (4.1) and (4.8) by the well-known procedure of envelope construction (5).

\section{The Linear Problem $u_{n}=h(s) u$}

Here the partial differential equation (2.9) reduces to

$$
u_{1} \tau_{u_{1}}+u_{2} \tau_{u_{2}}=0 \text {, }
$$

and signifies that $\tau$ is homogeneous of degree 0 in $u_{1}, u_{2}$, so we may write

$$
\tau=\tau(\lambda), \quad \lambda=u_{1} / u_{2},
$$

the function $\tau(\lambda)$ being arbitrary. On calculating $a, b, c$ from (2.3) we obtain

$$
a=\tau^{\prime}, \quad b=-\lambda \tau^{\prime}, \quad c=\lambda^{2} \tau^{\prime} .
$$

The simplest choice for the arbitrary function $\tau(\lambda)$ is $\tau=\lambda$, in which case the conditions (2.4) are clearly fulfilled, but to insure the validity of $(2.5)$ and thereby complete the hypothesis in Theorem 2.1 we make the additional assumption that the ratio $\lambda$ is regular analytic in $R$. Taking $u_{0}=0$ in Theorem 2.1 we arrive at the following theorem (4).

Theorem 5.1. If $u_{2}$ 丰 0 is a solution of the boundary problem

$$
\Delta u=0 \text { in } S, u_{n}=h(s) u \text { on } C \text {, }
$$

regular analytic in $R \supset S$, any other solution $u_{1}$ for which the ratio $\lambda=u_{1} / u_{2}$ is regular analytic in $R$ is linearly dependent on $u_{2}$.

6. The Non-linear Problems $u_{n}=h(s) u^{1+p}(p=1,2, \ldots)$

For the new variables $\xi, \eta$ in (3.9) we find

$$
\xi=\frac{1}{p}\left(\frac{1}{u_{2}^{p}}-\frac{1}{u_{1}^{p}}\right), \quad \eta=(1+p) \log |\lambda|, \quad \lambda=u_{1} / u_{2} .
$$


If $p$ is even, the inverse transformation is

$$
u_{1}^{p}=\frac{e^{g \eta}-1}{p \xi}, \quad u_{2}^{p}=\frac{1-e^{-\theta \eta}}{p \xi}, \quad g=\frac{p}{1+p},
$$

and the partial differential equation (3.9) becomes

$$
t_{\xi}+t t_{\eta}=\frac{g}{2} \operatorname{coth} \frac{g}{2} \eta \cdot t^{2}-\frac{t}{\xi} .
$$

This has the solution

$$
t=\frac{2 \gamma}{g} \frac{\sinh \frac{g}{2} \eta}{\xi}, \quad \gamma=\text { const., }
$$

as may be discovered by separation of variables or verified by direct substitution. Expressed in terms of the original variables this solution is

$$
t=\gamma(1+p)\left(u_{1} u_{2}\right)^{p / 2} \text {. }
$$

If $p$ is even, $t$ is regular analytic in $R$; if $p$ is odd we take

$$
t=\gamma(1+p)\left( \pm u_{1} u_{2}\right)^{p / 2} \text { as } u_{1} u_{2} \gtrless 0,
$$

and $t$ is regular analytic in $R$ provided neither $u_{1}$, nor $u_{2}$ vanishes in $R$. With this function $t$ at hand we turn to Theorem (3.1) and prove

Theorem 6.1. Two non-constant solutions $u_{1}, u_{2}$ of the boundary problem

$$
\Delta u=0 \text { in } S, \quad u_{n}=h(s) u^{1+p} \text { on } C, \quad p=1,2, \ldots
$$

regular analytic in $R$ with

$$
u_{1} \neq 0, \quad u_{2} \neq 0, \quad u_{1}^{p} \neq u_{2}^{p},
$$

holding in $R$ cannot exist.

To prove the theorem assume that two such solutions $u_{1}, u_{2}$ exist. If $p$ is even $f(u)=u^{1+p}$ is odd and Theorem 3.1. implies $u_{1}+u_{2}=0$ to contradict (6.3); if $p$ is odd, $f(u)=u^{1+p}$ is even and Theorem 3.1 implies $u_{1}=u_{2}$ again contradicting (6.3). Thus two such solutions $u_{1}, u_{2}$ cannot exist.

A variant of this theorem may be obtained by calculating $\tau$ explicitly. When $p$ is even from (3.4) and (6.2) we find

and therefore

$$
T_{\xi}=\frac{1-\gamma^{2}}{g \xi}, \quad T_{\eta}=\gamma \operatorname{csch} \frac{g}{2} \eta-\operatorname{coth} \frac{g}{2} \eta,
$$

so that

$$
T=\frac{1-\gamma^{2}}{g} \log \xi-\frac{4 \gamma}{g} \operatorname{arctanh} e^{g / 2 \eta}-\frac{2}{g} \log \sinh \frac{g}{2} \eta+\text { const., }
$$

$$
\tau=C_{0} \xi^{1-\gamma^{2} / g} e^{-4(\gamma / g) \text { arc tanh } e^{(g / 2) \eta}}\left(\sinh \frac{g}{2} \eta\right)^{-2 / g}, \quad C_{0}=\text { const. }
$$


To return to the original variables $u_{1}, u_{2}$ it is convenient to note from (6.1), (6.2) that

$$
e^{g \eta}=\lambda^{p}, \quad \xi=\frac{\lambda^{p}-1}{p u_{1}^{p}}, \quad \lambda=u_{1} / u_{2},
$$

whereupon after some calculation, we find

and from (2.3) that

$$
\tau=\lambda^{1+p} \Lambda u_{1}^{\left(\gamma^{2}-1\right)(1+p)}, \quad \Lambda=C \frac{e^{-4(\gamma / g) \text { arc tanh } \lambda p^{p / 2}}}{\left(1-\lambda^{p}\right)^{\left(\gamma^{2}+1\right) / g}}, \quad C=\text { const., }
$$

$$
\begin{array}{r}
a=(1+p) \frac{\left(\gamma-\lambda^{p / 2}\right)^{2}}{1-\lambda^{p}} \Lambda u_{1}^{2 p}, \quad b=-(1+p) \frac{\left(\gamma-\lambda^{p / 2}\right)\left(\gamma \lambda^{p / 2}-1\right)}{1-\lambda^{p}} \lambda^{1+p / 2} \Lambda u_{1}^{2 p}, \\
c=(1+p) \frac{\left(\gamma \lambda^{p / 2}-1\right)^{2}}{1-\lambda^{p}} \lambda^{2+p} \Lambda u_{1}^{2 p}, \quad \ldots \ldots .
\end{array}
$$

provided the arbitrary constant $\gamma$ is chosen so that $\gamma^{2}(1+p)=1+2 p$.

These formulas remain valid if $p$ is odd provided $0<\lambda<1$ and apply to prove the following theorem (4).

Theorem 6.2. If $u_{2}$ is a non-constant solution of the boundary problem

$$
\Delta u=0 \text { in } S, u_{n}=h(s) u^{1+p} \text { on } C . \quad p=1,2, \ldots,
$$

regular analytic in $R \supset S$, no other such solution $u_{1}$ exists for which the ratio $\lambda=u_{1} / u_{2}$ is regular analytic in $R$ and $|\lambda|<1$ if $p$ is even, or $0<\lambda<1$ if $p$ is odd.

If such a solution $u_{1}$ exists, $\tau$ is regular analytic in $R$, conditions (2.4) are met and (2.5) is valid, as is readily seen from (6.4) and (6.5). The conditions of Theorem 2.1 are therefore satisfied and this theorem implies $u_{1}=-u_{2}$ in $R$ if $p$ is even, and $u_{1}=u_{2}$ if $p$ is odd, contradicting the hypothesis on $\lambda$ in either case.

7. The Non-linear Problem $u_{n}=h(s) \sin u$

The solution is not unique. Given a solution $u_{1}$, other solutions $u_{2}$ are given by

$$
u_{2}=-u_{1}, \quad u_{2}=u_{1}+2 n \pi, \quad n=+1,+2, \ldots,
$$

but, under certain conditions the solution is unique, as we shall see in Theorem 7.1 below.

The transformation to new variables (3.8) gives

$$
\xi=\frac{1}{2} \log \frac{\tan ^{2} u_{1} / 2}{\tan ^{2} u_{2} / 2}, \quad \eta=\frac{1}{2} \log \frac{\sin ^{2} u_{1}}{\sin ^{2} u_{2}},
$$

with the inverse transformation

$$
\cos u_{1}=\frac{e^{\eta}-\cosh \xi}{\sinh \xi}, \quad \cos u_{2}=\frac{\cosh \xi-e^{-\eta}}{\sinh \xi},
$$

E.M.S. -C 
and the partial differential equation (3.9) takes the form (4.8) with

so that

$$
w=\frac{1}{2} \log \frac{\cosh \xi-\cosh \eta}{\sinh \xi},
$$

$$
Q=\sqrt{\frac{\sinh \xi}{\cosh \xi-\cosh \eta}}
$$

A simple calculation verifies that $Q$ satisfies conditions (4.11) with

and that the substitution

$$
\begin{aligned}
F(\xi) & =\frac{1}{4} \operatorname{csch}^{2} \xi . \\
z & =\operatorname{coth} \xi \ldots .
\end{aligned}
$$

transforms (4.10) into the equation

$$
\left(1-z^{2}\right) \ddot{Z}-2 z \dot{Z}-\frac{1}{4} Z=0, \quad \cdot=d / d z,
$$

for the Legendre functions of order $n=-\frac{1}{2}$. The general solution of this equation is (6)

$$
Z=c_{1} P_{-\frac{1}{2}}(z)+c_{2} Q_{-\frac{1}{2}}(z),
$$

and the Legendre functions may be represented by Laplace's integrals $P_{n}(z)=\frac{1}{\pi} \int_{0}^{\pi}\left(z+\sqrt{z^{2}-1} \cos \phi\right)^{n} d \phi, \quad Q_{n}(z)=\int_{0}^{\infty}\left(z+\sqrt{z^{2}-1} \cosh \theta\right)^{-n-1} d \theta$, of the first and second kind.

We take $c_{1}=\pi, c_{2}=0$ in (7.5), substitute for $z$ from (7.4), and obtain

$$
Z(\xi)=\int_{0}^{\pi} \sqrt{\frac{\sinh \xi}{\cosh \xi+\cos \phi}} d \phi, \quad H(\xi, \eta)=\int_{0}^{\eta} \sqrt{\frac{\sinh \xi}{\cosh \xi-\cosh \phi}} d \phi, \ldots
$$

the latter formula arising from (4.9) and (7:3). These functions may be expressed in terms of the elliptic integrals

$$
K(k)=\int_{0}^{\pi / 2} \frac{d \theta}{\sqrt{1-k^{2} \sin ^{2} \theta}}, \quad F(k, \phi)=\int_{0}^{\phi} \frac{d \theta}{\sqrt{1-k^{2} \sin ^{2} \theta}},
$$

if desired. One finds

$$
\begin{aligned}
& Z(\xi)=2 \sqrt{\tanh \frac{\xi}{2}} K(k), \quad k=\operatorname{sech} \frac{\xi}{2}, \\
& \xi, \eta)=2 \sqrt{\tanh \frac{\xi}{2}}[K(k)-F(k, \phi)], \quad k=\tanh \frac{\xi}{2}, \quad \sin \phi=\sqrt{\frac{\cosh \xi-\cosh \eta}{\cosh \xi-1}} .
\end{aligned}
$$

Clearly $Z, H$ are real, regular analytic functions of $\xi, \eta$ as long as

$$
0<|\eta|<\xi
$$

which condition will, from (7.1), be met if

$$
0<\left|u_{2}\right|<\left|u_{1}\right|<\pi \text {, }
$$


Since $Z \neq 0$ obviously holds for $\xi>0$, the solution $t$ of (4.8) given by (4.9) is regular analytic in the region (7.7) and consequently from (7.1) is regular analytic in the region (7.8). The following theorem follows from Theorem 3.1.

Theorem 7.1. Two solutions $u_{1}, u_{2}$ of the boundary problem

for which

$$
\Delta u=0 \text { in } S, \quad u_{n}=h(s) \sin u \text { on } C,
$$

hold in $R$ cannot exist.

$$
0<\left|u_{1}\right|<\left|u_{2}\right|<\pi
$$

For if two such solutions exist, conditions (3.7) are met and the solution $t$ of (3.4) regular analytic in $R$ is provided by (4.9) with $Z, H$ given in (7.6). The hypotheses of Theorem 3.1 are satisfied, and therefore either

$$
u_{2}-u_{1}=2 n \pi, \text { or } u_{2}+u_{1}=0
$$

holds, to contradict the hypothesis $0<\left|u_{1}\right|<\left|u_{2}\right|<\pi$.

\section{REFERENCES}

(1) T. Boggio, Sulle funzioni di variable complessa ui un'area circolari, Rendiconti della $R$. Accademia de Torino, 47 (1912), 22-37.

(2) A. Weinstern, Fluid motion with free boundaries, Proceedings of the First Symposium in Applied Mathematics, American Mathematical Society (1949), 1-18.

(3) J. J. STOKER, Water waves, Interscience (1957), 38-41.

(4) M. H. MARTIN, Linear and nonlinear boundary problems for harmonic functions, Proceedings American Mathematical Society, 10 (1959), 258-266.

(5) E. Goursat, Lecons sur l'intégration des équations aux dérivées partielles du premier ordre, Paris (1921), 134-153.

(6) E. T. Whittaker, A Course of Modern Analysis (Cambridge, 4th Edition, 1927), 302-319.

Institute for Fluid Dynamics and Applied Mathematics

UNIVERSITY OF MARYLAND

College Park, Maryland

U.S.A. 